\title{
Study of the Dressed State of Hydrogen Atom in Electron Atom Elastic Collision in Laser Field
}

\section{K. Yadav, S. P. Gupta and J. J. Nakarmi}

\section{Journal of Nepal Physical Society}

Volume 6, Issue 2, December 2020

ISSN: 2392-473X (Print), 2738-9537 (Online)

\section{Editors:}

Dr. Binod Adhikari

Dr. Bhawani Joshi

Dr. Manoj Kumar Yadav

Dr. Krishna Rai

Dr. Rajendra Prasad Adhikari

Mr. Kiran Pudasainee

JNPS, 6 (2), 149-157 (2020)

DOI: http://doi.org/10.3126/jnphyssoc.v6i2.34870

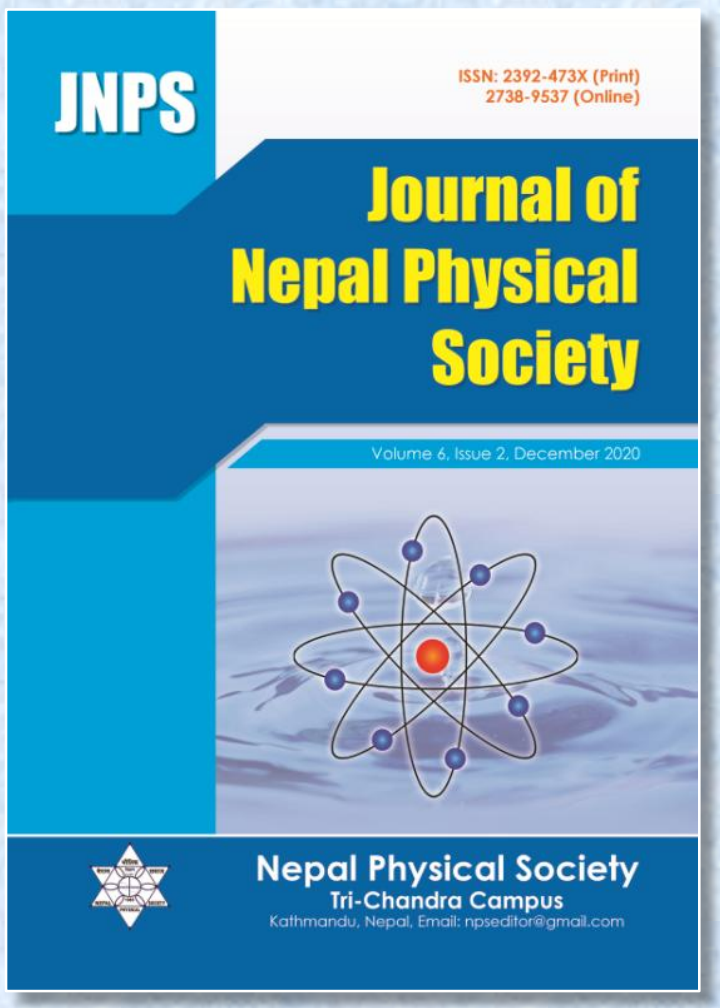

Published by:

Nepal Physical Society

P.O. Box: 2934

Tri-Chandra Campus

Kathmandu, Nepal

Email: npseditor@gmail.com 


\title{
Study of the Dressed State of Hydrogen Atom in Electron Atom Elastic Collision in Laser Field
}

\author{
K. Yadav ${ }^{1}$, , S. P. Gupta ${ }^{1}$ and J. J. Nakarmi ${ }^{2}$ \\ ${ }^{1}$ Patan Multiple Campus, Lalitpur, Tribhuvan University, Nepal \\ ${ }^{2}$ Central Department of Physics, Kirtipur, Tribhuvan University, Nepal \\ *Corresponding Email: yadavkishori70@gmail.com
}

Received: 19 October, 2020; Revised: 23 November, 2020; Accepted: 27 December, 2020

\begin{abstract}
We discuss the various important aspects of the theory of electron-atom elastic collision in laser field. We analyze the collision accompanied by the transfer of 1 photons. We study the free electron states i.e. Volkov states and target atomic states i.e. Dressed states. We calculate the first-Born scattering matrix for electron-atom elastic collision. The present work accounts the calculation for hydrogen as target atom in soft photon limits i.e., in weak field. The dressing effect becomes significant in the region of low momentum transfer, reaches maximum of value of 25000 a.u. at momentum transfer of 0.44 . This work explains that the differential crosssection does not occur very low and at very high momentum transfer. However, it occurs at moderate momentum transfer.
\end{abstract}

Keywords: Dressed state, Differential cross section, Scattering matrix, Scattering amplitudes.

\section{INTRODUCTION}

With the development of laser technology, the laser-assisted electron-atom (ion) scattering processes received considerable attention [1]. Due to their time-dependent nature, however, a unified treatment seems impossible. Various approximate methods are developed to deal with a certain aspect of the problem, depending on the specific parameters applied to the lasers and the atoms. Even for the simplest case of a Coulomb potential plus a time-dependent laser-electron interaction, the Schrodinger equation is not exactly solvable, although the solution for either individual potential is available. A product of both solutions for individual potential, called the Coulomb Volkov function, was suggested [2]. Such a solution is valid only when the ratio between the field strength and the field frequency is not so large.

In the recent years, the study of electron atom collision in the presence of a laser field is a subject of intense research activity, not only because the importance of these processes in applied domains (such as plasma heating), but also in view of their interest in fundamental atomic collision theory. Experimentally, laser-assisted electron-atom scattering processes have recently become feasible. Several experiments have been performed, in which the exchange of one or more photons between the electron target and the laser field has been observed in laser-assisted elastic and inelastic scattering [3]. The first theoretical studies on the inelastic scattering were inspired from the pioneering works $[4,5,6]$, in which the inter action between the free electron and the field can be treated exactly (i.e. to all orders in the field strength) by using the exact Volkov waves [2].

The theoretical study of electron-atom collisions in the presence of a laser field is in general very complex, since in addition to the difficulties associated with the treatment of field-free electronatom scattering, the presence of the laser field introduces new parameters (such as the laser frequency, intensity, polarization and statistics) which can influence the collisions. It is therefore of interest to begin the theoretical analysis by considering the simpler problem of the scattering of an electron by a potential, in the presence of a laser field. During the past decade, the availability of increasingly more powerful lasers has stimulated considerable interest in multi photon process. 
Among these process so called free-free transitions in which a charged particle (like electron) is scattered by an atomic target in presence of field.

When considering the collision of electron with real atom in presence of laser field, one has to take account three distinct interaction. First the interaction is between the unbound electron and the atomic system take place, as in the field free case. The second is radiation field interact strongly with unbound electron which induces inverse of Bremsstrahlung. Third is the modification of the atomic state by electromagnetic wave which can be represented by

$$
e^{-}+A^{*}+1 h \omega \rightarrow e^{-}+A^{*}+1^{\prime \prime} h \omega
$$

In such collision, the dressed states of the atoms $A$ in presence of laser field are closely coupled in the Volkov state of projectile electron in the laser field. The laser can perturb both the bound atomic electron as well as the incident projectile electron. The first effect was acknowledged by semiclassical Floquet approach [7]. The approach is compared to dressing by the traditional perturbation method which is shown to provide an incomplete description of the laser interaction and which can't predict the distinct features provided by the Floqute approach. The second effect of the laser interaction on the projectile electron is acknowledged via Volkov states.

In this work we have presented preliminary results showing that the dressing of the target produces important effects on the elastic scattering (accompanied by the transfer of 1 photons) of fast electrons by atoms in the presence of a laser field. These results were illustrated by calculations performed for an atomic hydrogen target in geometry such that the electric field was parallel to the momentum transfer. In the present work we will give a detailed derivation of final results and consider applications to elastic - atomic - hydrogen collision. We begin by discussing the lasermodified wave functions for the free electron and the target atom, the former is treated to all orders in the electron-laser-field interaction, while the latter is obtained to first order in the laser field, assuming that laser field is much less than the atomic unit of field strength. Then we consider successively the direct and exchange scattering amplitudes, using a perturbation treatment in the electron-atom interaction. The present work contains our results, obtained for an incident electron energy for lowfrequency lasers. In general, we find that the dipole distortion of the target produced by the laser field has a significant effect on the differential cross section at small momentum transfers, particularly when the number of exchanged photons is not equal to zero.

\section{MATERIAL AND METHOD}

\subsection{Dressed States of Hydrogen Atom}

When the atom is naked to the laser field, then the states of atom is said to be dressed states. The dressed states of the target atom embedded in the laser field are obtained by solving the Schrodinger equation

$$
i \hbar \frac{\partial}{\delta t} \phi\left(\overrightarrow{r_{1}}, \overrightarrow{r_{2}} \ldots . \overrightarrow{r_{z}}, t\right)=H_{T} \phi\left(\overrightarrow{r_{1}}, \overrightarrow{r_{2}} \ldots . \overrightarrow{r_{z}}, t\right)
$$

Where $\mathrm{z}$ is the atomic number of the atom and $\mathrm{H}_{\mathrm{T}}$ is the Hamiltonian of the target atom in the presence of laser field. We shall restrict our attention to laser assisted elastic scattering from the ground state of light atom such as atomic hydrogen where spin effect can be neglected.

$$
\begin{aligned}
& i \hbar\left(\frac { \partial } { \delta t } \left(\left(\overrightarrow{r_{1}}, \overrightarrow{r_{2}} \ldots . \overrightarrow{r_{z}}, t\right)=H_{T} \phi\left(\overrightarrow{r_{1}}, \overrightarrow{r_{2}} \ldots . \overrightarrow{r_{z}}, t\right)\right.\right. \\
& \text { or, } i \hbar\left(\frac{\partial}{\delta t}\left(\overrightarrow{r_{1}}, \overrightarrow{r_{2}} \ldots . \overrightarrow{r_{z}}, t\right)=\left[\frac{P^{2}}{2 m}-\frac{Z e^{2}}{r^{2}}+V_{e f}\right] \phi\left(\overrightarrow{r_{1}}, \overrightarrow{r_{2}} \ldots . \overrightarrow{r_{z}}, t\right)\right. \\
& \text { or, } i \hbar\left(\frac{\partial}{\delta t}\left(\overrightarrow{r_{1}}, \overrightarrow{r_{2}} \ldots . \overrightarrow{r_{z}}, t\right)=\left[\frac{P^{2}}{2 m}-\frac{Z e^{2}}{r^{2}}-\frac{e}{m c} \vec{A}(t) . \vec{P}+\frac{e^{2} A^{2}}{2 m c^{2}}\right] \phi\left(\overrightarrow{r_{1}}, \overrightarrow{r_{2}} \ldots . \overrightarrow{r_{z}}, t\right)\right. \\
& \text { or, } i \hbar\left(\frac{\delta}{\delta t}\left(\overrightarrow{r_{1}}, \overrightarrow{r_{2}} \ldots . \overrightarrow{r_{z}}, t\right)=\left[H_{e}+H^{\prime}\right] \phi\left(\overrightarrow{r_{1}}, \overrightarrow{r_{2}} \ldots . \overrightarrow{r_{z}}, t\right)\right.
\end{aligned}
$$

Since we are working on low frequency range, this energy associated with field can be neglected and we take $H^{\prime}=-\frac{e}{m c} \vec{A}(t) \cdot \vec{P}$ as the perturbation of the system due to presence of laser field.
The probability amplitude for the first order approximation is given by [8]

$b_{f}(t)=-\frac{1}{i \hbar} \int_{o}^{t} H_{f i}^{\prime}\left(\vec{r}, t^{\prime}\right) e^{i w_{f i} t^{\prime}} d t^{\prime}$ 
Where matrix element, $\mathrm{H}_{\mathrm{fi}}\left(\vec{r}, t^{\prime}\right)=\left\langle\psi_{f}\left|H^{\prime}\right| \psi_{i}\right\rangle$

Taking $\vec{A}(t)$ as linear harmonic, we have

$$
\vec{A}(t)=\vec{A}_{o} \varepsilon e^{i \omega t}+\vec{A}_{0} \varepsilon e^{-i \omega t} .
$$

Then, perturbation becomes

$$
H^{\prime}=\vec{A}_{o} \epsilon \cdot \vec{P} e^{i \omega t}+\vec{A}_{o} \epsilon \cdot \vec{P} e^{-i \omega t}
$$

We have,

$$
b_{f}(t)=-\frac{1}{i \hbar} \int_{o}^{t}\left\langle\psi_{f}^{(0)}\left|H^{\prime}\right| \psi_{i}^{(0)}\right\rangle e^{i w_{f i} t^{\prime}} d t^{\prime}
$$

$b_{f}(t)=-\frac{1}{i \hbar} \int_{o}^{t}\left\langle\vec{A}_{o} \epsilon \cdot \vec{P} e^{i \omega t^{\prime}} \mid i\right\rangle e^{i w_{f i} t^{\prime}} d t^{\prime}-\frac{1}{i \hbar} \int_{o}^{t}\left\langle f\left|\vec{A}_{o} \epsilon \cdot \vec{P} e^{-i \omega t^{\prime}}\right| i\right\rangle e^{i w_{f i} t^{\prime}} d t^{\prime}$

$b_{f}(t)=-\frac{1}{i \hbar} \int_{o}^{t}\left\langle f\left|\vec{A}_{o} \epsilon \cdot \frac{1}{2 i}\left[r, H_{0}\right]\right| i\right\rangle e^{i \omega t^{\prime}} \cdot e^{i w_{f i} t^{\prime}} d t^{\prime}-\frac{1}{i \hbar} \int_{o}^{t}\left\langle f<\vec{A}_{o} \epsilon \cdot \frac{1}{2 i}\left[r, H_{0}\right] \mid i\right\rangle e^{-i \omega t^{\prime}} \cdot e^{i w_{f i} t^{\prime}} d t^{\prime}$

Where $\vec{P}=m r^{\cdot}=\frac{1}{i \hbar}\left[r, H_{0}\right]$ is equation of motion and its real value $\frac{1}{2}<\left[r, H_{0}\right]>$ and $\frac{1}{2}$ is introduced to take real expectation value

$b_{f}(t)=\frac{1}{i \hbar} \int_{o}^{t}\left(E_{i}-E_{f}\right)\left\langle f\left|\vec{A}_{o} \epsilon \cdot \vec{r}\right| i\right\rangle \int_{o}^{t} e^{-i\left(w+w_{f i}\right) t^{\prime}} d t^{\prime}+\frac{1}{i \hbar}\left(E_{i}-E_{f}\right)\left\langle f\left|\vec{A}_{o} \epsilon \cdot \vec{r}\right| i\right\rangle \int_{o}^{t} e^{i\left(w_{f i}-w\right) t^{\prime}} d t^{\prime}$

On integration, we have

$b_{f}(t)=\frac{1}{i \hbar} \int_{o}^{t}\left(E_{i}-E_{f}\right)\left\langle f\left|\vec{A}_{o} \epsilon \cdot \vec{r}\right| i\right\rangle\left[\frac{e^{i\left(\omega+\omega_{f i}\right) t}}{\left(\omega+\omega_{f i}\right)}-\frac{e^{i\left(\omega_{f i}-\omega\right) t}}{\left(\omega_{f i}-\omega\right)}\right]$

This expression gives a peak value at different condition. The second terms gives a peak value at $w_{f i}=w$, i.e. $w_{f}-w_{i}=w$

For resonant absorption, $E_{f}>E_{i}$ i.e. The first term gives the peak value at $w_{f i}=-w$ i.e. $w_{f}-w_{i}=$ $-w$ which implies that $E_{i}>E_{f}$ i.e. stimulated emission.
Now, we know from first order time dependent perturbation theory, the wave function of the dressed state is given by

$\phi(\vec{r}, t)=\sum_{n^{\prime}} b_{f}^{\prime}(t) \varphi_{n}^{(0)} e^{\frac{-i E n t}{\hbar}}$

Using the value of $b_{f}(t)$ for $\mathrm{m}^{\text {th }}$ state

$\phi_{m}(\vec{r}, t)=e^{-i \omega_{m n} t} e^{-\vec{a} \cdot \vec{R}}\left[\psi_{m}(r)+\frac{1}{2 \hbar} \sum_{m}\left(\frac{e^{i w t}}{w_{m n}+w}-\frac{e^{-i w t}}{w_{m n}-w}\right) \cdot M_{m n} \psi_{m n}(r)\right.$

Where $\psi_{m}(\vec{r})$ is the unperturbed atomic wave function (Target state of energy in absence of laser field ) with energy $E_{m}=\mathrm{h} \omega_{m}$ and $\omega_{m n}=\omega_{m}-\omega_{n}=$ Bohr frequency and $M_{m n}=\vec{E}_{0} \cdot\left\langle\psi_{m}|\vec{E} \cdot \vec{r}| \psi_{n}\right\rangle$ is dipole matrix element and $\overrightarrow{\mathrm{EO}}$ is the atomic field strength less than $5 \times 10^{9} \mathrm{~V} / \mathrm{C}$ and summation includes an integration over the continuum states. The factor $e^{\vec{a} \cdot \vec{R}}$ ensures gauge consistency between the Volvok free wave function. This wave function is valid for all frequency $\boldsymbol{\omega}$ except in the vicinity of a Bohr's frequency and $\psi_{m}(\vec{r})$ (unperturbed wave function) is added because it is spherical symmetry. So, the ground state of dressed wave function is given by

$\phi n(\vec{r}, t)=e^{-i \omega_{0} t} e^{-\vec{a} \cdot \vec{R}}\left[\psi n(r)+\sum_{n^{\prime}}\left(\frac{e^{i w t}}{w_{n 0}+w}-\frac{e^{-i w t}}{w_{n 0}-w}\right) \psi n(r) M n 0\right.$

\subsection{Direct scattering amplitude and area of differential cross-sectional}

The S-matrix element for elastic scattering in the direct channel, in the presence of laser field and in the first Born approximation is given as [6]
$S_{e l}^{B 1}=-i \int_{-\infty}^{\infty} d t\left\langle\chi_{k f}(\vec{r} O, t) \phi_{0}(\vec{r}, t)|V d| \chi_{k i}\right.$

$\left.(\vec{r} o, t) \phi_{0}(\vec{r}, t)\right\rangle$ 
Where $V d=-\frac{z}{r_{0}}+\sum_{J=1}^{z} \frac{1}{r_{0 j}}$ is the direct electro- $\quad \chi_{k f}=(2 \pi)^{\frac{-3}{2}} \exp \left[i\left(\vec{k}_{f} \cdot \vec{r}-\vec{k}_{f} \cdot \alpha \sin \omega t-\frac{E_{f} t}{\hbar}\right)\right]$ atom interaction potential; with $\overrightarrow{r_{o j}}=\left|\overrightarrow{r_{0}}-\overrightarrow{r_{j}}\right|$ and $\overrightarrow{r_{0}}$ denotes the co-ordinate of projectile electron. Here $\overrightarrow{k_{l}}$ and $\overrightarrow{k_{f}}$ denote respectively the wave vectors of the free - electron projectile before and $\left.\chi_{k i}=(2 \pi)^{\frac{-3}{2}} \exp \left(\mathrm{i} \vec{k}_{i} \cdot \vec{r}-\vec{k}_{i} \cdot \alpha \sin \omega t-\frac{E_{i} t}{\hbar}\right)\right]$ after the scattering then the Volkov state wave function is given by,

Using equation (18) and (19) in equation (17) we get

$S_{e l}^{B 1}=-i \int_{-\infty}^{+\infty} d t \int(2 \pi)^{\frac{-3}{2}} e^{\left[i\left(\vec{k}_{f}-\vec{k}_{i}\right) \cdot \vec{r}+\left(\vec{k}_{f}-\vec{k}_{i}\right) \cdot \vec{\alpha} \sin \omega t \frac{\left(E_{f-} E_{i}\right) t}{\hbar}\right]} *\left\langle\phi_{0}(r, t)|V d| \phi_{0}(r, t)\right\rangle d r_{o}$

We define, $\Delta=\left|\vec{k}_{f}-\vec{k}_{i}\right|$ is the momentum transfer and also $x \cong \Delta . \vec{\alpha}$

$S_{e l}^{B 1}=-\int_{-\infty}^{+\infty} d t \int(2 \pi)^{-3} e^{i\left(\Delta \cdot \vec{r}_{0}\right)} e^{i x \sin \omega t} e \frac{\left(E_{f}-E_{i}\right) t}{\hbar} d r_{o} \cdot\langle\phi o(\vec{r}, t)|V d| \phi 0(\vec{r}, t)\rangle$

Using the generating function for Bessel function

$e^{(i x \sin u)}=\sum_{l=-\infty}^{\infty} J_{l}(x) \exp (i l u)$

$S_{e l}^{B 1}=-\int_{-\infty}^{+\infty} d t \int(2 \pi)^{-3} e^{i\left(\Delta \cdot \vec{r}_{0}\right)} e \frac{\left(E_{f}-E_{i}\right) t}{\hbar} d r_{o} \sum_{l=-\infty}^{\infty} J_{l}(x) \exp (i l w t) X\left\langle\phi o(\vec{r}, t)|V d| \phi_{0}(\vec{r}, t)\right\rangle$

$S_{e l}^{B 1}=-\int_{-\infty}^{+\infty} d t \int(2 \pi)^{-3} \sum_{=-\infty}^{\infty} e \frac{\left(E_{f}-E_{i}-l \hbar \omega\right) t}{\hbar} J_{l}(X) \int e^{i\left(\Delta \cdot \vec{r}_{0}\right)} d r_{o}\left\langle\phi_{o}(\vec{r}, t)\left|V_{d}\right| \phi_{0}(\vec{r}, t)\right\rangle$

Suppose

$\mathrm{T}_{l}^{(\mathrm{o})}=\sum_{\mathrm{l}=-\infty}^{+\infty} \mathrm{J}_{\mathrm{l}}(\mathrm{x}) \widetilde{\mathrm{V}}(\Delta)\left\langle\phi \mathrm{o}(\overrightarrow{\mathrm{r}}, \mathrm{t})|\mathrm{Vd}| \phi_{0}(\overrightarrow{\mathrm{r}}, \mathrm{t})\right\rangle$

$=\widetilde{\mathrm{V}}(\Delta) \sum_{\mathrm{l}=-\infty}^{+\infty} \mathrm{J}_{\mathrm{l}}(\Delta \cdot \vec{\alpha})\left\langle\phi o(\overrightarrow{\mathrm{r}}, \mathrm{t})|\mathrm{Vd}| \phi_{0}(\overrightarrow{\mathrm{r}}, \mathrm{t})\right\rangle$

where,

$\widetilde{\mathrm{V}}(\Delta)=(2 \pi)^{-3} \int \mathrm{e}^{\mathrm{i}\left(\Delta \cdot \overrightarrow{\mathrm{r}}_{0}\right)} \mathrm{dr}_{\mathrm{o}}$

Therefore

$S_{e l}^{B 1}=-i \mathrm{~T}_{\mathrm{l}}^{(\mathrm{o})} \int_{-\infty}^{+\infty} d t e \frac{\left(E_{f}-E_{i}-l \hbar \omega\right) \mathrm{t}}{\hbar}$

$S_{e l}^{B 1}=-i \mathrm{~T}_{1}^{(0)} \int_{-\infty}^{+\infty} e \frac{\left(E_{f}-E_{i}-l \hbar \omega\right) \mathrm{t}}{\hbar} d t$

(20)
So this S-Matrix element related to the transition amplitude for transition from momentum state $\mathrm{K}_{\mathrm{i}}$ to $\mathrm{K}_{\mathrm{f}}$ and $\mathrm{T}_{\mathrm{l}}^{(\mathrm{o})}$ is the transition matrix which is time independent. So it is taken outside from the time integration.

Also

$\mathrm{T}_{1}^{(\mathrm{o})}=\mathrm{J}_{1}(\Delta)\left\langle\phi \mathrm{o}(\overrightarrow{\mathrm{r}}, \mathrm{t})|\widetilde{\mathrm{V}} \mathrm{d}| \phi_{0}(\overrightarrow{\mathrm{r}}, \mathrm{t})\right\rangle$

In circular polarization, it relates to the BunkinFreedov formula in which dressing effect of the target is neglected and Bessel function contents all the field dependences of the transition matrix element and the form factor operator $\widetilde{\mathrm{V}}_{\mathrm{d}}(\Delta)$ is given by (9)

$\widetilde{\mathrm{V}}_{\mathrm{d}}(\Delta)=\frac{1}{2 \pi^{2} \Delta^{2}} \exp ([\mathrm{i} \Delta \cdot \overrightarrow{\mathrm{r}}-1])$ 
Using direct delta function equation (20)

$S_{e l}^{B 1}=-2 \pi i \mathrm{~T}_{\mathrm{l}}^{(\mathrm{o})} \delta\left(E_{f}-E_{i}-l \hbar \omega\right)$

Then, the differential cross-sectional area is given by

$\frac{d \sigma}{d \Omega}=\frac{m^{2}}{(2 \pi)^{2} h^{4}} \frac{k_{f}}{k_{i}}\left|T_{l}^{o}\right|^{2}$

$\frac{d \sigma}{d \Omega}=\frac{m^{2}}{(2 \pi)^{2} h^{4}}\left(1-\frac{l h w}{E_{k i}}\right)^{1 / 2}\left|T_{l}^{o}\right|^{2}$
Where $\frac{k_{f}}{k_{i}}=\left(1-\frac{l h w}{E_{k i}}\right)^{1 / 2}$ and

$E_{k i}$ is initial kinetic energy

When the dressing effect is taken into account then the dressing effects of the atomic target is called first order correction to the atomic state. One of the 1 photons exchange between the field and colliding system interacting with the bound electron and is calculated using dressed state wave function at ground state of equation (16) we get

$\phi_{0}\left(\overrightarrow{r_{0}}, t\right)=\mathrm{e}^{-\mathrm{i} \omega \mathrm{t}} e^{-i \vec{a} \cdot \vec{R}}\left[\psi_{0}\left(\vec{r}_{0}\right)-\operatorname{sinwt} \sum_{n} \frac{w_{n o} M_{n o}}{w_{n 0}^{2}-w^{2}}-i \cos w t \sum_{n} \frac{w M_{n o}}{w_{n 0}^{2}-w^{2}} \psi n\left(\vec{r}_{0}\right)\right.$

Where, $\psi n\left(\vec{r}_{0}\right)$ is the target state of energy $\mathrm{w}_{\mathrm{no}}$ in the absence of laser field. $\mathrm{W}_{\mathrm{no}}=\left(\mathrm{E}_{\mathrm{n}}-\mathrm{E}_{\mathrm{o}}\right)$ is the Bohr's frequency and $\mathrm{M}_{\mathrm{no}}<\psi_{n}\left|\epsilon E_{0} \cdot \vec{R}\right| \psi_{0}$ is the dipole coupling Matrix element. Using equation (23) in equation (17) and on calculation we get,

$$
\begin{array}{rl}
S_{e l}^{B 1}=-(2 \pi)^{-1} & i \sum_{l=\infty}^{-\infty} \delta\left(E_{f}-E_{i}-l \hbar \omega\right)\left[<\psi_{0}\left(r_{0}\right)|V d| \psi_{0}\left(r_{0}\right)\right. \\
& >-\sin w t \sum_{n} \frac{w_{n o} M_{n o}}{w_{n 0}^{2}-w^{2}}<\psi_{0}|V d| \psi_{n}>-i \cos w t \sum_{n} \frac{W_{n o} M_{n o}}{w_{n 0}^{2}-w^{2}}<\psi_{0}|V| \psi_{n} \\
& \left.>-\sin w t \sum_{n}^{n} \frac{w_{n o} M_{n o}}{w_{n 0}^{2}-w^{2}}<\psi_{n}|V d| \chi_{0}>-i \cos w t \sum_{n} \frac{w_{n o} M_{n o}}{w_{n 0}^{2}-w^{2}}<\psi_{n}\left|V_{d}\right| \chi_{0}>\right]
\end{array}
$$

Taking the Fourier transform,

$$
\int \exp \left(i \Delta . \vec{r}_{0}\right) V d\left(\vec{r}_{0}, x\right) d r_{0}=\frac{4 \pi}{\Delta^{2}} V d(\Delta, x)
$$

We get,

$S_{e l}^{B 1}=$

$-2 \pi i \sum_{l=-\infty}^{\infty} \delta\left(E_{f}-E_{i}-l \hbar \omega\right) J_{l}(x) \frac{-2}{\Delta^{2}}\left[\left\langle\psi_{0}\left(r_{0}\right)\left|\widetilde{\mathrm{V}}_{\mathrm{d}}\right| \psi_{0}\left(r_{0}\right)\right\rangle-\sin \omega t \sum_{n} \frac{\omega_{n 0}}{\omega_{n 0}{ }^{2}-\omega^{2}} \frac{-2}{\Delta^{2}}\left\langle\psi_{0}\left|\widetilde{\mathrm{V}}_{\mathrm{d}}\right| \psi_{n}\right\rangle-\right.$ $i \cos \omega t \sum_{n} \frac{\omega M_{n 0}}{\omega_{n 0^{2}}{ }^{2} \omega^{2}} \frac{-2}{\Delta^{2}}\left\langle\psi_{n}\left|\widetilde{V}_{\mathrm{d}}\right| \psi_{0}\right\rangle-\sin \omega t \sum_{n} \frac{\omega_{n 0} M_{n 0}}{\omega_{n 0}{ }^{2}-\omega^{2}} \frac{-2}{\Delta^{2}}\left\langle\psi_{n}\left|\widetilde{V}_{\mathrm{d}}\right| \psi_{0}\right\rangle-$

$\left.i \cos \omega t \sum_{n} \frac{\omega M_{n 0}}{\omega_{n 0}{ }^{2}-\omega^{2}} \frac{-2}{\Delta^{2}}\left\langle\psi_{n}\left|\widetilde{V}_{\mathrm{d}}\right| \psi_{0}\right\rangle\right]$

$\therefore \quad S_{e l}^{B 1}=-2 \pi i \sum_{l=-\infty}^{\infty}\left(E_{f}-E_{i}-l \hbar \omega\right) J l(x) \frac{-2}{\Delta^{2}}\left[\left\langle\psi_{0}\left(r_{0}\right)\left|\widetilde{\mathrm{V}}_{\mathrm{d}}\right| \psi_{0}\right\rangle\right.$

$-J l(x) \sin \omega t \sum_{n} \frac{\omega_{n 0} M_{n 0}}{\omega_{n 0^{2}-\omega^{2}}} \cdot \frac{-2}{\Delta^{2}}\left\langle\psi_{0}\left|\widetilde{\mathrm{V}}_{\mathrm{d}}\right| \psi_{n}\right\rangle-i \cos \omega t J l(x) \sum_{n} \frac{\omega_{n 0}}{\omega_{n 0^{2}-\omega^{2}}} \frac{-2}{\Delta^{2}}\left\langle\psi_{0}\left|\widetilde{\mathrm{V}}_{\mathrm{d}}\right| \psi_{n}\right\rangle-$

$\left.J l(x) \sin \omega t \sum_{n} \frac{\omega_{n 0} M_{n 0}}{\omega_{n 0}{ }^{2}-\omega^{2}}\left(\frac{-2}{\Delta^{2}}\right)\left\langle\psi_{n}\left|\widetilde{V}_{\mathrm{d}}\right| \psi_{0}\right\rangle-i \cos \omega t J l(x) \sum_{n} \frac{w_{n o} M_{n 0}}{\omega_{n 0}{ }^{2}-\omega^{2}} \frac{-2}{\Delta^{2}}\left\langle\psi_{n}\left|\widetilde{V}_{\mathrm{d}}\right| \psi_{0}\right\rangle\right]$

where,

$\exp (\mathrm{iySinu})=\sum_{\mathrm{l}=-\infty}^{\infty} \mathrm{Jl}(\mathrm{y}) \exp (\mathrm{ilu})$

$$
S_{e l}^{B 1}=-2 \pi i \sum_{l=-\infty}^{\infty} \delta\left(E_{f}-E_{i}-\hbar \omega\right)\left[J l ( x ) \frac { - 2 } { \Delta ^ { 2 } } \left[\left\langle\psi_{0}\left(r_{0}\right)\left|\widetilde{\mathrm{V}}_{\mathrm{d}}\right| \psi_{0}\right\rangle\right.\right.
$$




$$
\begin{aligned}
& -i \sin \omega t \exp (i x \operatorname{Sinwt}) \exp (-i l w t) \sum_{n} \frac{\omega_{n 0} M_{n 0}}{\omega_{n 0}^{2}-\omega^{2}}\left(\frac{-2}{\Delta^{2}}\right)\left\langle\psi_{0}\left|\widetilde{V}_{\mathrm{d}}\right| \psi_{n}\right\rangle \\
& -i \operatorname{Sin} \omega t \exp (i x \operatorname{Sin} w t) \exp (-i l w t) \sum_{n} \frac{\omega_{n 0}}{\omega_{n 0}^{2}-\omega^{2}}\left(\frac{-2}{\Delta^{2}}\right)\left\langle\psi_{n}\left|\widetilde{V}_{\mathrm{d}}\right| \psi_{0}\right\rangle
\end{aligned}
$$

Where cos term is imaginary, so neglected.

$$
\begin{aligned}
& \therefore \quad S_{e l}^{B 1}=2 \pi i \sum_{l=-\infty}^{\infty} \delta(E f-E i-l \hbar \omega)\left[J _ { l } ( \frac { - 2 } { \Delta ^ { 2 } } ) \left[\left\langle\psi_{0}\left(r_{0}\right)\left|\widetilde{V}_{\mathrm{d}}\right| \psi_{0}\right\rangle\right.\right. \\
& -i J_{l}^{\prime}(x) \sum_{n} \frac{\omega_{n 0}}{\omega_{n 0^{2}}{ }^{2} \omega^{2}}\left(\frac{-2}{\Delta^{2}}\right)\left\langle\psi_{0}\left|\widetilde{V}_{\mathrm{d}}\right| \psi_{n}\right\rangle x M_{o n}-i J_{l}^{\prime} \sum_{n} \frac{\omega_{n 0}}{\omega_{n 0^{2}-\omega^{2}}}\left(\frac{-2}{\Delta^{2}}\right)\left\langle\psi_{n}\left|\widetilde{V}_{\mathrm{d}}\right| \psi_{0}\right\rangle M_{n o} \\
& \therefore \quad S_{e l}^{B 1}=-2 \pi i \sum_{l} \delta\left(E_{f}-E_{i}-l \hbar \omega\right)\left[\mathrm{T}_{\mathrm{fi}}^{\mathrm{o}}(\Delta)-i J_{l}^{\prime}(x) \sum_{n} \frac{\omega_{n 0}}{\omega_{n 0}^{2}-\omega^{2}}\left(\mathrm{~T}_{o, n p}^{B_{1}}(\Delta)+\mathrm{T}_{n p, 0}^{B_{1}}(\Delta)\right)\right.
\end{aligned}
$$

$\mathrm{T}_{n p, 0}^{B_{1}}(\Delta)=-\frac{2}{\Delta^{2}}\left\langle\psi_{n}\left|\widetilde{\mathrm{V}}_{\mathrm{d}}\right| \psi_{0}\right\rangle$

Are the Born amplitude corresponding to the scattering amplitude 0 to $\mathrm{np}$ and $\mathrm{np}$ to 0 transition in the absence of laser field and sum involves only intermediate $\mathrm{P}$ states, therefore transition $\operatorname{matrixT}_{f i}^{2}(\Delta)$ is given by

$$
\mathrm{T}_{f i}^{2}(\Delta)=\mathrm{T}_{f i}^{0}(\Delta)+\mathrm{T}_{f i}^{1}(\Delta)
$$

$$
\emptyset_{0}(r, t)=e^{+i \omega_{0} t} \cdot e^{i \vec{a} \cdot \vec{r}} \cdot\left[\psi_{0}(r)-\sin \omega t \widetilde{\psi_{n}}-i \cos \omega t \widetilde{\psi_{n}}\right]
$$

Same as Volkov wave function $\left(\mathrm{eq}^{\mathrm{n}}(17)\right)$, then calculation becomes,

$$
\begin{aligned}
& S_{e l}^{B 1}=-2 \pi i \sum_{i=-\infty}^{\infty} \delta\left(E_{f}-E_{i}-l \hbar \omega\right) J l(x)\left(\frac{-2}{\Delta^{2}}\right)\left[\left\langle\psi_{0}\left(r_{0}\right)\left|\widetilde{\mathrm{V}}_{\mathrm{d}}\right| \psi_{0}\right\rangle-\right. \\
& \sin w t\left(\frac{-2}{\Delta^{2}}\right)\left\langle\psi_{0}\left|\widetilde{\mathrm{V}}_{\mathrm{d}}\right| \psi_{n}\right\rangle-i \cos w t\left(\frac{-2}{\Delta^{2}}\right)\left\langle\psi_{0}\left|\widetilde{\mathrm{V}}_{\mathrm{d}}\right| \psi_{n}\right\rangle-\sin w t\left(\frac{-2}{\Delta^{2}}\right)\left\langle\psi_{n}\left|\widetilde{\mathrm{V}}_{\mathrm{d}}\right| \psi_{0}\right\rangle-i \cos w t\left(\frac{-2}{\Delta^{2}}\right)\left\langle\psi_{n}\left|\widetilde{\mathrm{V}}_{\mathrm{d}}\right| \psi_{0}\right\rangle
\end{aligned}
$$

After neglecting imaginary terms,.

$$
\begin{aligned}
& S_{e l}^{B 1}=-2 \pi i \sum_{l=-\infty}^{\infty} \delta\left(E_{f}-E_{i}-l \hbar \omega\right)\left[J l ( x ) \frac { - 2 } { \Delta ^ { 2 } } \left[\left\langle\psi_{0}\left(r_{0}\right)\left|\widetilde{\mathrm{V}}_{\mathrm{d}}\right| \psi_{0}\right\rangle\right.\right. \\
& -i \sin \omega t \exp (i x \operatorname{Sin} w t) \exp (-i l w t)\left(\frac{-2}{\Delta^{2}}\right)\left\langle\psi_{0}\left|\widetilde{V}_{\mathrm{d}}\right| \psi_{n}\right\rangle \\
& -i \operatorname{Sin} \omega t \exp (i x \operatorname{Sin} w t) \exp (-i l w t)\left(\frac{-2}{\Delta^{2}}\right)\left\langle\psi_{n}\left|\widetilde{V}_{\mathrm{d}}\right| \psi_{0}\right\rangle \\
& \therefore S_{e l}^{B 1}=-2 \pi i \sum_{l=-\infty}^{\infty} \delta\left(E_{f}-E_{i}-l \hbar \omega\right) \mathrm{T}_{\mathrm{fi}}^{2}(\Delta)
\end{aligned}
$$


where,

$\mathrm{T}_{f i}^{2}(\Delta)=J_{l}(x) \mathrm{T}_{f i}^{0}(\Delta)-i J_{l}^{\prime}(x) f^{B l}\left(\tilde{\psi}_{0} \rightarrow \psi_{0}\right)+$ $\left.f^{B l}\left(\psi_{0} \rightarrow \tilde{\psi}_{0}\right)\right)$

Here $f^{b l}$ denotes $T_{0, n p}^{B 1}$ and $T_{n p, 0}^{B 1}$ i.e. Born amplitude.

Where the first terms represent the field amplitude and second term is just the first Born corresponding to transition from the state to the state or vice-versa. For the case of an atomic hydrogen target a closed form expansion can be calculated to $\widetilde{\psi}_{0}$. For $\tilde{\psi}_{0}$, the Hamiltonian of hydrogen atom is denoted by $h=\frac{P^{2}}{2}-\frac{1}{r}$ then receiving energy is given for one photon

$\left(h-w_{0}\right) \tilde{\psi}_{0}(r)=e \vec{E}_{o} \cdot \vec{r} \psi_{0}(r)$

We know the ground state wave function of hydrogen atom,

$\psi_{0}(r)=\pi^{-1 / 2} e^{-r}$

Solution of the equation can be written as

$\tilde{\psi}_{0}=\pi^{-\frac{1}{2}} e \vec{E}_{o} r\left(1+\frac{\vec{r}}{2} \exp (r)\right.$

Therefore, we have [11]

$$
\mathrm{T}_{f i}^{0}(\Delta)=-\frac{2}{\Delta^{2}}\left\langle\tilde{\psi}_{0}\left|\widetilde{\mathrm{V}}_{\mathrm{d}}\right| \psi_{n}\right\rangle=\frac{2\left(\Delta^{2}+8\right)}{\left(\Delta^{2}+4\right)^{2}}
$$

Similarly,

$$
\begin{gathered}
f_{\left(\psi_{0} \rightarrow \widetilde{\psi}_{0}\right)}^{B l}+f_{\left(\widetilde{\psi}_{0 \rightarrow} \psi_{0}\right)}^{B l}=2 \bar{\Delta}^{2}\left\langle\tilde{\psi}_{0}\left|\widetilde{V}_{\mathrm{d}}\right| \psi_{n}\right\rangle=\frac{192 E_{o}}{\Delta^{2}\left(\Delta^{2}+4\right)}\left(1+\frac{8}{\left(\Delta^{2}+4\right)}\right) \\
=T_{n p, 0}^{1}+T_{0, n p}^{1}
\end{gathered}
$$

Again from [12]

$$
\mathrm{T}_{f i}^{2}(\Delta)=J_{l}(x) \frac{2\left(\Delta^{2}+8\right)}{\left(\Delta^{2}+4\right)^{2}}-J_{l}^{\prime}\left(\frac{192 E_{o} \cdot \Delta}{\Delta^{2}\left(\Delta^{2}+4\right)^{3}}\left(1+\frac{8}{\left(\Delta^{2}+4\right)}\right)\right.
$$

Differential cross-section area in dressed state of Hydrogen atom for the net exchange of 1 photons between the colliding atomic system and laser field is given as

$$
\begin{aligned}
& \frac{d \sigma}{d \Omega}=\frac{m^{2}}{(2 \pi)^{2} h^{4}}\left(1-\frac{l h w}{E_{k i}}\right)^{1 / 2} \mathrm{~T}_{f i}^{2}(\Delta) \\
& \frac{d \sigma}{d \Omega}=\frac{m^{2}}{(2 \pi)^{2} h^{4}}\left(1-\frac{l h w}{E_{k i}}\right)^{1 / 2}\left[J_{l}(x) \frac{2\left(\Delta^{2}+8\right)}{\left(\Delta^{2}+4\right)^{2}}-J_{l}^{\prime}\left(\frac{192 E_{o} . \Delta}{\Delta^{2}\left(\Delta^{2}+4\right)^{3}}\left(1+\frac{8}{\left(\Delta^{2}+4\right)}\right)\right]^{2} .\right.
\end{aligned}
$$

\section{RESULTS AND DISCUSSION}

\subsection{Differential cross-section}

Let us analyze the result obtained in previous section, for weak field the scattering amplitude is given by,

$$
\begin{aligned}
\mathrm{T}_{f i}^{2}(\Delta)=J_{l}(x) & \frac{2\left(\Delta^{2}+8\right)}{\left(\Delta^{2}+4\right)^{2}} \\
& -J_{l}^{\prime}\left(\frac{192 E_{o} . \Delta}{\Delta^{2}\left(\Delta^{2}+4\right)^{3}}\left(1+\frac{8}{\left(\Delta^{2}+4\right)}\right)\right.
\end{aligned}
$$

For $J_{1}(x)$ is ordinary Bessel function $\mathrm{J}^{\prime}(\mathrm{x})$ is its derivatives, $1<0$ for emission and $1>0$

For absorption, $\Delta=\overrightarrow{K_{f}}-\overrightarrow{K_{\iota}}$ is momentum transfer which is less than one for weak field,
$\mathrm{x}=\Delta \cdot \alpha, \alpha=4.5$ for hydrogen, $\mathrm{E}_{0}=20$ a.u.

From recurrence relation (20)

$J_{l}^{\prime}(x)=\frac{l}{x} J_{1}(x)-J_{l+1}(x)$

$\mathrm{T}_{f i}^{2}(\Delta)=$

$J_{1}(x) \frac{2\left(\Delta^{2}+8\right)}{\left(\Delta^{2}+4\right)^{2}}-\frac{l}{x} J_{1}(x)-J_{l+1}(x) \frac{192 \vec{E}_{0} . \Delta}{\Delta^{2}\left(\Delta^{2}+4\right)^{3}}(1+$

$\left.\frac{8}{\Delta^{2}+4}\right)(29)$

The first terms is the field free scattering amplitudes $\left(\mathrm{T}_{f i}^{0}(\Delta)\right)$ with ordinary Bessel function. The second terms account the dressing effects. For the simplicity we choose variable $\mathrm{x}$ as a function of momentum transfer i.e. $x=4.5 \Delta$. For target atoms value of $\alpha$ is 
taken as constant. The value of $\mathrm{x}$ decreases as momentum transfer decreases. The second terms with ordinary Bessel function will significant for small value of $\mathrm{x}$. From the first Born scattering amplitude, the differential cross section is given as

$$
\frac{d \sigma}{d \omega}=|f(\theta)|^{2}
$$

The plots of differential cross section against the momentum transfer using computational program has been plotted as shown in Fig, 1 for $1=1$ i.e. in the case of photon absorption.

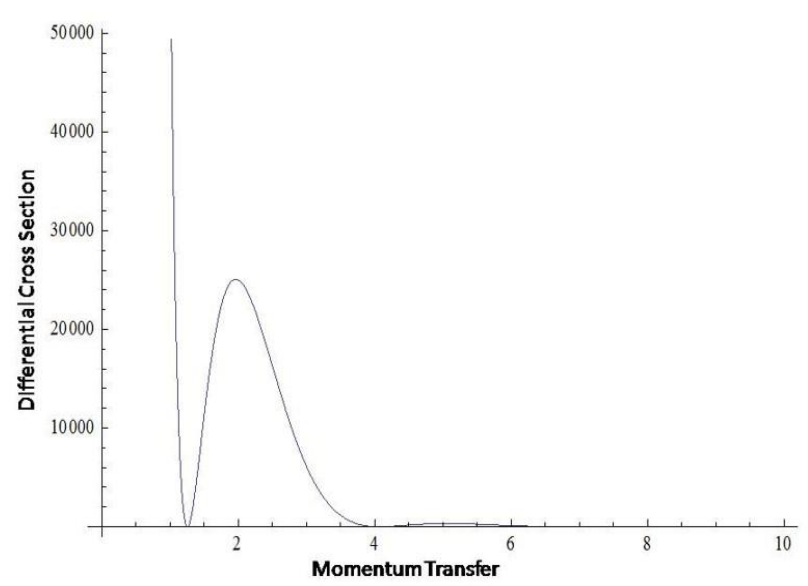

Fig. 1: Differential cross section against momentum transfer

Figure 1 explains that below momentum transfers 0.4 , the differential cross-sectional area increases in large amount such that particles diverge due to large area of cross-section at small momentum transfer which blocks the particles. In the above plot resonance occurs at momentum transfer 0.44 which cross ponds to the absorption of photon and this absorption cross-sectional area fits for this range of momentum transfer.

When contribution of momentum transfer is very small the differential cross section is large. In figure 2 the range of momentum transfer is very low therefore particles get diverge because of large cross-sectional area.

\section{CONCLUSIONS}

In present work we studied the electron atom elastic collision in weak laser field, which is accompanied by the momentum transfer. We discuss the free electron states as Volkov states and calculate the Volkov wave functions.

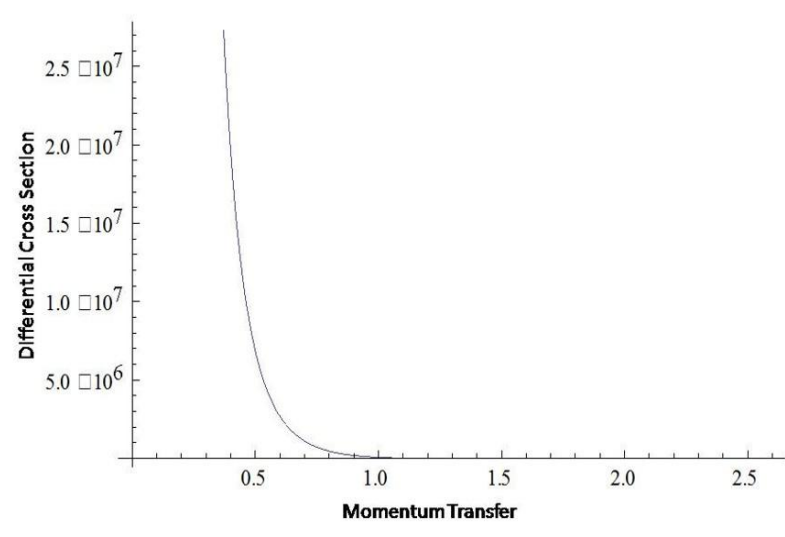

Fig.2: Differential cross section against momentum transfer.

We also analyzed the target atom is dress by laser field and whose solution is given by dressed states wave functions. This works gave a detail derivation of the first Born scattering matrix for electron atom elastic collisions in presence of weak field i.e., in soft photon limits. We found scattering matrix contains energy terms and amplitudes terms. There were several amplitudes, the field free amplitude and amplitudes for various transitions. In this work we analyzed that the dressing of target produces important effects on electron atom elastic collisions. The dressing effects became more significant in the region of low momentum transfer. In the high momentum transfer region, the dressing effect became less significant and almost absent. The dressing effect causes important modification in differential cross-section.

For the deferential cross section against momentum transfer at $\mathrm{x}=4.5 \Delta$, the Fig. 2 showed that deferential cross section approaches to infinite since dressing effect is significant in the region of low momentum transfer. Indeed, the second term in the equation (29) containing $\frac{l}{x}$ which became infinite. The initial falls of differential cross section with momentum transfer is due to the absorption of photons. Here probability of collision becomes less, and differential cross section approaches to lower value and then it increases and reached to the maximum value of $\approx 25000$ a.u. at momentum transfer of 0.44. In higher momentum transfer region, the dressing effect became less or in very high region it is almost absent. Fig.2. showed that the differential cross section for very small momentum transfer is very large. The differential cross-section occurs at moderate momentum transfer. 


\section{REFERENCES}

[1] Mittleman, M. H. Introduction to the theory of laser atom interaction, Plenum, New York (1993).

[2] Volkov, D. M. On a class of solutions of the Dirac equation, Z. Phys, 94(3-4): 250-260 (1935).

[3] Weingart shofer, A.; Holmes, J. K.; Caudle, G.; Clarke, E. M. and Krüger, H. Direct Observation of Multiphoton processes in Laser-Induced FreeFree Transitions, Phys. Rev. Lett. 39: 269 (1977).

[4] Byron, F. W.; Franckenand, P.; Joachain, C. J. Laser assisted elastic electron atom collision, $J$. Phys: B: At. Mol. Phys.,20: 5487-5503 (1987).

[5] Francken, P.; Attaourti, Y.; and Joachain, C. J. Laser assisted electron atom collision, Phys. Rev.A 38: 1785 (1988).

[6] Philippeand, F.; Joachain, C. J. Electron-atomichydrogen elastic collision in the presence of a laser field, Phys. Rev. A, 35(4): 1590 (1987).

[7] Dorr, M. and Joachain, C. J. Born-Floquet theory of laser-assisted electron-atom collisions Phys. Rev A. 49(6): (1994).
[8] Agrawal, B. K. and Prakash, H. Quantum Mechanics, Prentic Hall of India, New Delhi (1997).

[9] Buică, G. Polarization dependence in inelastic scattering of electrons by hydrogen atoms in a circularly polarized laser field, Journal of Quantitative Spectroscopy and Radiative Transfer, 187: 190-203 (2016).

[10] Yadav, K. and Nakarmi, J. J. Free-free scattering theory of the elastic scattering of an electron, International journal of physics, 3: 32-39 (2015).

[11] Yadav, K.; Nakarmi, J. J. and Maharjan, S. Free scattering theory in circularly polarized laser field, Journal of Nepal physical Society, 4(1): 78-87 (2017).

[12] Byron, F. W.; Francken, Jr, P. and Joachain, C. J. Laser-assisted elastic electron-atom collisions. Journal of Physics B: Atomic and Molecular Physics, 20(20): 5487 (1987). 